\title{
Computation of the mid-sagittal plane in diffusion tensor MR brain images
}

\author{
Sylvain Prima ${ }^{a, b, c, d}$ and Nicolas Wiest-Daesslé $a, b, c, d$ \\ ${ }^{a}$ INRIA, VisAGeS U746 Unit/Project, IRISA, Campus de Beaulieu, F-35042 Rennes, France \\ ${ }^{b}$ University of Rennes I, IRISA, Campus de Beaulieu, F-35042 Rennes, France \\ ${ }^{c}$ CNRS, IRISA UMR 6074, Campus de Beaulieu, F-35042 Rennes, France \\ ${ }^{d}$ INSERM, VisAGeS U746 Unit/Project, IRISA, Campus de Beaulieu, F-35042 Rennes, France
}

\begin{abstract}
We propose a method for the automated computation of the mid-sagittal plane of the brain in diffusion tensor MR images. We estimate this plane as the one that best superposes the two hemispheres of the brain by reflection symmetry. This is done via the automated minimisation of a correlation-type global criterion over the tensor image. The minimisation is performed using the NEWUOA algorithm in a multiresolution framework. We validate our algorithm on synthetic diffusion tensor MR images. We quantitatively compare this computed plane with similar planes obtained from scalar diffusion images (such as FA and ADC maps) and from the B0 image (that is, without diffusion sensitisation). Finally, we show some results on real diffusion tensor MR images.
\end{abstract}

Keywords: Registration, optimisation methods, diffusion tensor imaging, brain symmetry, mid-sagittal plane.

\section{INTRODUCTION}

The human brain displays an approximately bilateral symmetry with respect to the mid-sagittal plane (MSP). This gross symmetry is often partially hidden in medical images, when the head is scanned in a tilted position. In this situation, homologous bilateral structures (ventricles, grey nuclei, etc.) do not appear on the same 2D axial or coronal slices, which can lead to misinterpretation of the images. Having a coherent display of medical images with respect to the cerebral MSP can be useful for many applications.

First, it allows to remove the inter-hemispheric differences only due to the head tilt. After correction of the tilt, the remaining normal or abnormal asymmetries can be assessed qualitatively or quantitatively, which can be relevant for many pathologies. Second, the MSP itself is a crucial landmark for further analyses, such as morphometry of the corpus callosum, delineation of the AC-PC line, or global quantification of brain symmetries and asymmetries. Third, knowing the position of this plane is useful in registration tasks, where such an a priori knowledge about the images to be matched can improve the robustness, accuracy and computation speed of the methods.

Many algorithms have been developed for the automated computation of the cerebral mid-sagittal plane in medical images. Two different approaches have been mainly used. Some works define the mid-sagittal plane as the plane that best matches the inter-hemispheric fissure. ${ }^{1-4}$ Such techniques are only applicable when this fissure and its characteristic features are clearly visible on images, limiting their application to MR and CT images. A more general approach is to view the mid-sagittal plane as the one that best separates the brain into two similar parts. Many methods have been developed based on this idea, dealing with either anatomical (MR and CT) or functional images (PET, SPECT). ${ }^{5-12}$

Diffusion Tensor Magnetic Resonance Imaging (DT-MRI or DTI for short) complements conventional, anatomical, MR imaging in that it adds specific structural information about the brain. By measuring diffusion properties of water molecules in the brain, it allows the indirect visualisation of the microscopic organisation of tissues, and more especially the white matter. There is an increasing interest in how DTI conveys brain symmetry and asymmetry. ${ }^{13-20}$

Further author information: (Send correspondence to Sylvain Prima) Sylvain Prima: E-mail: sprima@irisa.fr, Telephone: 33299847359

Medical Imaging 2007: Image Processing, edited by Josien P. W. Pluim, Joseph M. Reinhardt, Proc. of SPIE Vol. 6512, 65121I, (2007) · 1605-7422/07/\$18 · doi: 10.1117/12.709467 
In this paper, we propose a method for the automated computation of the mid-sagittal plane of the brain in DTI. We estimate this plane as the one that best superposes the two hemispheres of the brain by reflection symmetry. This is done via the automated minimisation of a correlation-type global criterion over the image data, i.e. diffusion tensors. The minimisation is performed using the NEWUOA algorithm in a multiresolution framework.

Such an algorithm has the same potential applications as the methods developed for anatomical and functional images. More specifically, it has a direct application on DTI visualisation, which is often a critical task due to the complex, non-scalar, nature of the data under study. For example, the classical red-green-blue colormap applied to the principal direction of diffusion for easier display of DTI (red for left-right, green for antero-posterior and blue for top-bottom $)^{21}$ is of poor interest if the mid-sagittal plane (and thus the left-right direction) is not aligned with the image grid.

We detail our method in Section 2. We make a quantitative evaluation of its robustness and accuracy properties, and present some results on real data in Section 3. We conclude and give some perspectives in Section 4.

\section{METHOD}

\subsection{Data acquisition}

Images were obtained on a whole-body Philips 3T MR scanner. A single-shot echo-planar sequence combined with coil sensitivity encoding (SENSE) was used to acquire the diffusion-weighted MR images with a spin-echo Stejskal-Tanner sequence $\left(\mathrm{TE} / \mathrm{TR}(\mathrm{ms})=56 / 7991\right.$, flip angle $\left.=90^{\circ}\right) \cdot{ }^{22}$ For each subject, an image without diffusion sensitisation was acquired ( $\mathrm{b}$ value $=0 \mathrm{sec} / \mathrm{mm}^{2}$ ), together with diffusion-weighted MR images (b value $=800 \mathrm{sec} / \mathrm{mm}^{2}$ ) with diffusion-sensitising gradients applied in 16 different directions. The characteristics of the diffusion-weighted MR images are: matrix size $=256 \times 256$, pixel size $(\mathrm{mm})=0.875 \times 0.875$, FOV $(\mathrm{mm})=$ $224 \times 224 \times 120$, number of slices $=60$, slice thickness $(\mathrm{mm})=2$.

\subsection{Pre-processing}

The pre-processing stage includes the following steps:

- Correction of distortions. Diffusion-weighted images acquired with echo-planar sequences are significantly distorted, mainly due to the eddy currents induced by the large diffusion gradients. To deal with this artefact, each diffusion-weighted image was registered to the image without diffusion sensitisation. The alignment is based on the maximisation of a similarity measure on image intensities (the local correlation) and a transformation model based on the imaging physics. ${ }^{23}$

- Computation of a brain mask as the intersection of individual brain masks computed on each diffusionweighted MR image by histogram analysis. ${ }^{24}$

- Estimation of the diffusion tensor by multiple linear regression on a voxel-by-voxel basis. ${ }^{25}$

- Diagonalisation of the diffusion tensor by classical linear algebra (computation of the eigenvalues and eigenvectors).

- Computation of different quantitative MR diffusion parameters and related 3D maps: Apparent Diffusion Coefficient (ADC), Fractional Anisotropy (FA), Relative Anisotropy (RA), direction of the eigenvector with the largest eigenvalue. 


\subsection{Computation of the mid-sagittal plane}

\subsubsection{Some notations}

- $I$ is a diffusion tensor image, defined on a regular grid $G$

- For the sake of simplicity and without loss of generality we assume that $G$ is isotropic with voxel size 1 $\mathrm{mm}^{3}$

- $v$ is a voxel on the grid $G$, and its value $I(v)$ is a tensor (symmetric positive definite (SPD) $3 \times 3$ matrix)

- $a(b)$ is the value of the function $a$ for argument $b$, whereas $a b$ is the product of matrices $a$ and $b$; in the following, $v$ is considered as either the argument of a function or as a column vector, depending on the context

- $S_{P}$ is the reflection symmetry with respect to the plane $P$

- $M^{T}$ is the transpose of matrix $M$

- $x, y$ and $z$ are the left-right, antero-posterior and top-bottom axes, respectively

\subsection{Formulation of the problem}

If the brain was perfectly symmetrical, there would exist a symmetry plane $P$ superposing each voxel $v$ of the grid with its anatomical counterpart. If we note $v^{\prime}=S_{P}(v)$, it means that the tensors $I(v)$ and $I\left(v^{\prime}\right)$ would be mirror-symmetrical with respect to $P$, which writes:

$$
I(v)=S_{P}\left(I\left(v^{\prime}\right)\right)
$$

In real life, the brain is only grossly symmetrical, and such an ideal symmetry plane does not exist. Instead, we define the approximate symmetry (or mid-sagittal) plane $P$ as the plane minimising the average distance between the tensor $I(v)$ and the reflection of $I\left(v^{\prime}\right)$ with respect to $P$, where $v^{\prime}=S_{P}(v)$ is the voxel homologous to $v$ in the contralateral hemisphere. This average distance would be null for a perfect symmetry plane. The optimisation problem can then be written as:

$$
\tilde{P}=\underset{P}{\arg \min } \frac{1}{\operatorname{card}\left(G_{P}\right)} \sum_{v \in G_{P}} d\left(I(v), S_{P}\left(I\left(v^{\prime}\right)\right)\right), \quad \text { where: }
$$

- $v^{\prime}=S_{P}(v)$

- $G_{P}$ is the overlapping area between the two sets of voxels to compare: $G_{P}=G \cap S_{P}(G)$

- $d(.,$.$) is a metric on tensors$

\subsection{Implementation details}

\subsubsection{Parameterisation of $P$}

Any plane in $\mathbb{R}^{3}$ can be characterised by a normal unit vector $n$ and its distance $d$ to the origin. The unit vector $n$ is characterised by its angles $\alpha$ and $\beta$ in a spherical coordinate system, where:

- $\alpha$ is the latitude angle (the equator is in the $x y$-plane)

- $\beta$ is the longitude angle (the prime meridian is in the $x z$-plane) 
The coordinates of $n$ are:

$$
n^{T}=\left[\begin{array}{lll}
\cos (\beta) \cos (\alpha) & \sin (\beta) \cos (\alpha) & \sin (\alpha)
\end{array}\right]
$$

If we note $H=I d-2 n n^{T}$ ( $I d$ is the $3 \times 3$ identity matrix) and $t=2 d n$, then it can be easily shown that the reflection of the voxel $v$ and of the tensor $I(v)$ with respect to $P$ are equal to, respectively:

$$
S_{P}(v)=H v+t \quad \text { and } \quad S_{P}(I(v))=H I(v) H^{T}=H I(v) H, \text { as } H=H^{T}
$$

\subsubsection{Interpolation scheme}

For an arbitrary plane $P$, the voxel $v^{\prime}=S_{P}(v)$ does not necessarily coincide with a grid point of $G$. Thus, an interpolation is needed to estimate the tensor value $I\left(v^{\prime}\right)$ at this non-grid point. A natural choice would be to interpolate in log-Euclidean or affine-invariant frameworks, which would preserve the constraint of symmetric positive definiteness. ${ }^{26-29}$ This unfortunately leads to a high computational cost and a prohibitive computation time for the overall algorithm. Our choice is to simply use the nearest neighbor interpolation instead, which also guarantees that the interpolated value is a SPD matrix.

\subsubsection{Distance function}

The distance function $d$ is simply based on the Frobenius norm. For two tensors $C$ and $D, d$ is defined as:

$$
d(C, D)=\sqrt{\sum_{i=1}^{3} \sum_{j=1}^{3}\left(C_{i j}-D_{i j}\right)^{2}}
$$

\subsubsection{Optimisation}

We choose the algorithm NEWUOA (NEW Unconstrained Optimisation Algorithm) to solve the optimisation problem. This algorithm, originally proposed by M.J.D. Powell, ${ }^{30}$ has been shown to be significantly more accurate, more robust and faster than other deterministic, derivative-free algorithms such as Nelder-Mead's downhill simplex or Powell's direction set algorithms for intensity-based rigid-body image registration. ${ }^{31}$ The three parameters of interest are $\alpha, \beta$ and $d$ (that define the plane $P$ and thus the associated reflection symmetry $S_{P}$ ). The algorithm is initialised with a plane at the centre of the image grid, orthogonal to the left-right axis.

A multiresolution (simple decimation) approach is implemented to increase the robustness, accuracy and speed of the algorithm. A subsampled image is built by taking only one voxel out of $f_{x}$ (resp. $f_{y}, f_{z}$ ) in the $x$ - (resp. $y-, z-)$ direction in the original image. A first solution is found at this low resolution, and is used to initialise the algorithm at a higher resolution, and so on. In this coarse-to-fine scheme, typically two or three levels can be used, depending on the resolution of the original image.

\subsubsection{Realignment of the plane}

Once the MSP is estimated, it is realigned at the centre of the image grid for improved image display. ${ }^{7}$ Such a realignment is performed by applying the rigid transformation $R$ to both the anatomical and the tensor information. ${ }^{32}$ The rigid transformation $R$ is computed as:

$$
R=\left(S_{K} \circ S_{P}\right)^{-1 / 2}
$$

where $S_{P}$ is the reflection symmetry with respect to the estimated mid-sagittal plane and $S_{K}$ is the reflection symmetry with respect to $K$, the plane in the middle of the image grid. Let $r$ be the rotation part of $R$. Each tensor $I(v)$ of the image $I$ is rotated following: $R(I(v))=r I(v) r^{T}$. The log-Euclidean interpolation ${ }^{28}$ is used for computation of the realigned DTI. The overall computation time for a two-level multiresolution scheme is about 13 minutes for a typical DT image (matrix size $256 \times 256$ and 60 slices) on a standard PC (OS Linux), with an Intel Xeon $4 \mathrm{CPU}$ at $2.8 \mathrm{GHz}, 2$ GBytes of RAM. 


\section{VALIDATION AND RESULTS}

A synthetic dataset composed of 400 DTI is generated as explained in Fig. 1 for assessment of robustness and accuracy properties of our algorithm. We use the measures $\delta$ and $\epsilon$ to evaluate the capture range (i.e. the maximal tilt that it is able to correct) and the accuracy (i.e. the closeness between the estimated plane and the ground truth plane) of the algorithm, as shown in Fig. 2. The algorithm fails $(\epsilon>1 \mathrm{~mm})$ only for 14 out of 400 random DTI. The algorithm never fails when $\delta<43 \mathrm{~mm}$. The first mistake occurs for an experiment where $\delta=43.82 \mathrm{~mm}$, which corresponds to $\left(t_{x}, \phi_{y}, \phi_{z}\right)=(-18.26,18.45,2.68)$. These parameters are rather extreme and even unrealistic for real DTI, showing that our algorithm is robust to strong initial tilts (see Fig. 3). For the successful experiments $(\epsilon \leq 1 \mathrm{~mm})$, the RMS over the set of $\epsilon$ values is $0.11 \mathrm{~mm}$, showing that the algorithm reaches a subvoxel accuracy when it converges properly.

The algorithm achieves good performances in terms of robustness and accuracy. However, one could ask whether computing a MSP on scalar diffusivity/anisotropy maps extracted from the tensor image would give similar results with a highly reduced computation time. The same question could be asked for the corresponding ("B0") diffusion-unweighted image. To investigate this question, we have modified the algorithm to make it suitable for scalar images. The modified criterion (to be maximised in this case) is simply the mutual information between the image $I$ and its reflection with respect to $S_{P}$, computed over their overlapping area. The mutual information is estimated via the joint histogram of the two images, computed with a partial volume interpolation scheme.

The comparisons with the DTI-based MSP computation are led as follows. First, using the same dataset as in Fig. 1a, the B0 image and the ADC/FA maps are manually aligned with the centre of the image and half of each image is removed to give three perfectly symmetrical images, aligned with the synthetic DTI of Fig. 1b. Second, the same 400 random transformations are then applied to each of these three images, and the MSP is computed with the modified algorithm describes above. Three paired t-tests are then used to compare the accuracy $(\epsilon$ values) of the MSP computed in each of these scalar images with the MSP computed in the corresponding DTI. It appears that the latter is significantly more accurate than the three other ones $\left(p<5.10^{-4}\right)$. However, the computation of the MSP in these three scalar images is much faster and seems as robust as the computation of the MSP in the DTI. Additional experiments should be performed, with realistic noise simulation for each of the 400 generated datasets. In this case, we expect the MSP computation to be more robust in case of tensor data.

At last, we show some results on real DTI from patients with Parkinson's disease in Fig. 4.

\section{CONCLUSION}

In this paper, we have proposed a method for the automated computation of the mid-sagittal plane of the brain in diffusion tensor MR images. We demonstrated that it is both robust and accurate on a synthetic dataset. Moreover, we introduced a new optimisation algorithm (NEWUOA) to deal with the high computational burden of DTI data. The realignment of this plane improves the display of DTI data. In the future, we plan to experiment other implementation choices and compare them with the present method. The most straightforward modifications could be made in the choice of the interpolation scheme and of the distance function. Other minimisation techniques could also be explored, to avoid local minima of the criterion. We will also evaluate the robustness and accuracy properties of the algorithm in presence of image artefacts (noise, intensity inhomogeneities) and brain normal and pathological asymmetries.

\section{ACKNOWLEDGEMENTS}

Nicolas Wiest-Daesslé is supported by a grant from the ARSEP (Association pour la Recherche sur la Sclérose En Plaques: French association for research on multiple sclerosis).

\section{REFERENCES}

1. M. Brummer, "Hough transform detection of the longitudinal fissure in tomographic head images," IEEE Transactions on Medical Imaging 10, pp. 74-81, Mar. 1991. 
2. R. Guillemaud, P. Marais, A. Zisserman, B. McDonald, T. Crow, and M. Brady, "A three dimensional mid sagittal plane for brain asymmetry measurement," Schizophrenia Research 18, pp. 183-184, Feb. 1996.

3. P. Anbazhagan, A. Carass, P.-L. Bazin, and J. Prince, "Automatic estimation of midsagittal plane and AC-PC alignment based on nonrigid registration," in 3rd IEEE International Symposium on Biomedical Imaging: From Nano to Macro, ISBI'06, (Arlington, USA), Apr. 2006.

4. I. Volkau, K. N. B. Prakash, A. Ananthasubramaniam, A. Aziz, and W. L. Nowinski, "Extraction of the midsagittal plane from morphological neuroimages using the Kullback-Leibler's measure," Med Image Anal 10, pp. 863-874, Dec. 2006.

5. S. Smith and M. Jenkinson, "Accurate robust symmetry estimation," in Second International Conference on Medical Image Computing and Computer-Assisted Intervention, MICCAI'99, C. Taylor and A. Colchester, eds., Lecture Notes in Computer Science 1679, pp. 308-317, Springer, (Cambridge, UK), Sept. 1999.

6. Y. Liu, R. Collins, and W. Rothfus, "Robust midsagittal plane extraction from coarse, pathological 3D images," in Third International Conference on Medical Image Computing and Computer-Assisted Intervention, MICCAI'2000, S. Delp, A. DiGioia, and B. Jaramaz, eds., Lecture Notes in Computer Science 1935, pp. 83-94, Springer, (Pittsburgh, USA), Oct. 2000.

7. S. Prima, S. Ourselin, and N. Ayache, "Computation of the mid-sagittal plane in 3-D brain images.," IEEE Trans Med Imaging 21, pp. 122-138, Feb. 2002.

8. A. Tuzikov, O. Colliot, and I. Bloch, "Evaluation of the symmetry plane in 3D MR brain images," Pattern Recognition Letters 24, pp. 2219-2233, Oct. 2003.

9. S. Joshi, P. Lorenzen, G. Gerig, and E. Bullitt, "Structural and radiometric asymmetry in brain images," Medical Image Analysis 7, pp. 155-170, June 2003.

10. Q. Hu and W. Nowinski, "A rapid algorithm for robust and automatic extraction of the midsagittal plane of the human cerebrum from neuroimages based on local symmetry and outlier removal," NeuroImage 20, pp. 2153-2165, Dec. 2003.

11. M. Stegmann, K. Skoglund, and C. Ryberg, "Mid-sagittal plane and mid-sagittal surface optimization in brain MRI using a local symmetry measure," in Proceedings of SPIE Medical Imaging 2005: Image Processing, J. Reinhardt and J. Fitzpatrick, eds., pp. 568-579, SPIE Press, (San Diego, USA), Feb. 2005.

12. L. Teverovskiy and Y. Liu, "Truly 3D midsagittal plane extraction for robust neuroimage registration," in 3rd IEEE International Symposium on Biomedical Imaging: From Nano to Macro, ISBI'06, (Arlington, USA), Apr. 2006.

13. S. Peled, H. Gudbjartsson, C. F. Westin, R. Kikinis, and F. A. Jolesz, "Magnetic resonance imaging shows orientation and asymmetry of white matter fiber tracts," Brain Res 780, pp. 27-33, Jan. 1998.

14. Y. Cao, S. Whalen, J. Huang, K. L. Berger, and M. C. DeLano, "Asymmetry of subinsular anisotropy by in vivo diffusion tensor imaging," Hum Brain Mapp 20, pp. 82-90, Oct. 2003.

15. S. Zhang, M. E. Bastin, D. H. Laidlaw, S. Sinha, P. A. Armitage, and T. S. Deisboeck, "Visualization and analysis of white matter structural asymmetry in diffusion tensor MRI data," Magn Reson Med 51, pp. 140-147, Jan. 2004.

16. H.-J. Park, C.-F. Westin, M. Kubicki, S. E. Maier, M. Niznikiewicz, A. Baer, M. Frumin, R. Kikinis, F. A. Jolesz, R. W. McCarley, and M. E. Shenton, "White matter hemisphere asymmetries in healthy subjects and in schizophrenia: a diffusion tensor MRI study," Neuroimage 23, pp. 213-223, Sept. 2004.

17. C. Buechel, T. Raedler, M. Sommer, M. Sach, C. Weiller, and M. A. Koch, "White matter asymmetry in the human brain: a diffusion tensor MRI study," Cereb Cortex 14, pp. 945-951, Sept. 2004.

18. G. Gong, T. Jiang, C. Zhu, Y. Zang, F. Wang, S. Xie, J. Xiao, and X. Guo, "Asymmetry analysis of cingulum based on scale-invariant parameterization by diffusion tensor imaging," Hum Brain Mapp 24, pp. 92-98, Feb. 2005.

19. H. W. R. Powell, G. J. M. Parker, D. C. Alexander, M. R. Symms, P. A. Boulby, C. A. M. Wheeler-Kingshott, G. J. Barker, U. Noppeney, M. J. Koepp, and J. S. Duncan, "Hemispheric asymmetries in language-related pathways: a combined functional MRI and tractography study," NeuroImage 32, pp. 388-399, Aug. 2006.

20. T. R. Barrick, I. N. Lawes, C. E. Mackay, and C. A. Clark, "White matter pathway asymmetry underlies functional lateralization," Cereb Cortex, 2007. 
21. S. Pajevic and C. Pierpaoli, "Color schemes to represent the orientation of anisotropic tissues from diffusion tensor data: application to white matter fiber tract mapping in the human brain," Magn Reson Med 42, pp. 526-540, Sept. 1999.

22. T. Jaermann, G. Crelier, K. P. Pruessmann, X. Golay, T. Netsch, A. M. C. van Muiswinkel, S. Mori, P. C. M. van Zijl, A. Valavanis, S. Kollias, and P. Boesiger, "SENSE-DTI at 3 T," Magn Reson Med 51, pp. 230-236, Feb. 2004.

23. T. Netsch and A. van Muiswinkel, "Quantitative evaluation of image-based distortion correction in diffusion tensor imaging," IEEE Trans Med Imaging 23, pp. 789-798, July 2004.

24. N. Otsu, "A threshold selection method from grey scale histogram," IEEE Transactions on Systems, Man and Cybernetics 1, pp. 62-66, 1979.

25. P. Basser and D. Jones, "Diffusion-tensor MRI: theory, experimental design and data analysis - a technical review," NMR in Biomedicine 15, pp. 456-467, Nov-Dec 2002.

26. P. T. Fletcher and S. C. Joshi, "Principal geodesic analysis on symmetric spaces: statistics of diffusion tensors," in Computer Vision and Mathematical Methods in Medical and Biomedical Image Analysis, ECCV'2004 workshops CVAMIA and MMBIA, M. Sonka, I. A. Kakadiaris, and J. Kybic, eds., Lecture Notes in Computer Science 3117, pp. 87-98, Springer, (Prague, Czech Republic), May 2004.

27. P. G. Batchelor, M. Moakher, D. Atkinson, F. Calamante, and A. Connelly, "A rigorous framework for diffusion tensor calculus," Magnetic Resonance in Medicine 53, pp. 221-225, Jan. 2005.

28. V. Arsigny, P. Fillard, X. Pennec, and N. Ayache, "Log-Euclidean metrics for fast and simple calculus on diffusion tensors," Magnetic Resonance in Medicine 56, pp. 411-421, Aug. 2006.

29. X. Pennec, P. Fillard, and N. Ayache, "A Riemannian framework for tensor computing," International Journal of Computer Vision 66, pp. 41-66, Jan. 2006.

30. M. Powell, "The NEWUOA software for unconstrained optimization without derivatives," in 40th Workshop on Large Scale Nonlinear Optimization, Springer, (Erice, Italy), June 2004.

31. N. Wiest-Daesslé, P. Yger, S. Prima, and C. Barillot, "Evaluation of a new optimisation algorithm for rigid registration of MRI data," in Proceedings of SPIE Medical Imaging 200\%: Image Processing, 2007.

32. D. C. Alexander, C. Pierpaoli, P. J. Basser, and J. C. Gee, "Spatial transformations of diffusion tensor magnetic resonance images," IEEE Transactions on Medical Imaging 20, pp. 1131-1139, Nov. 2001. 


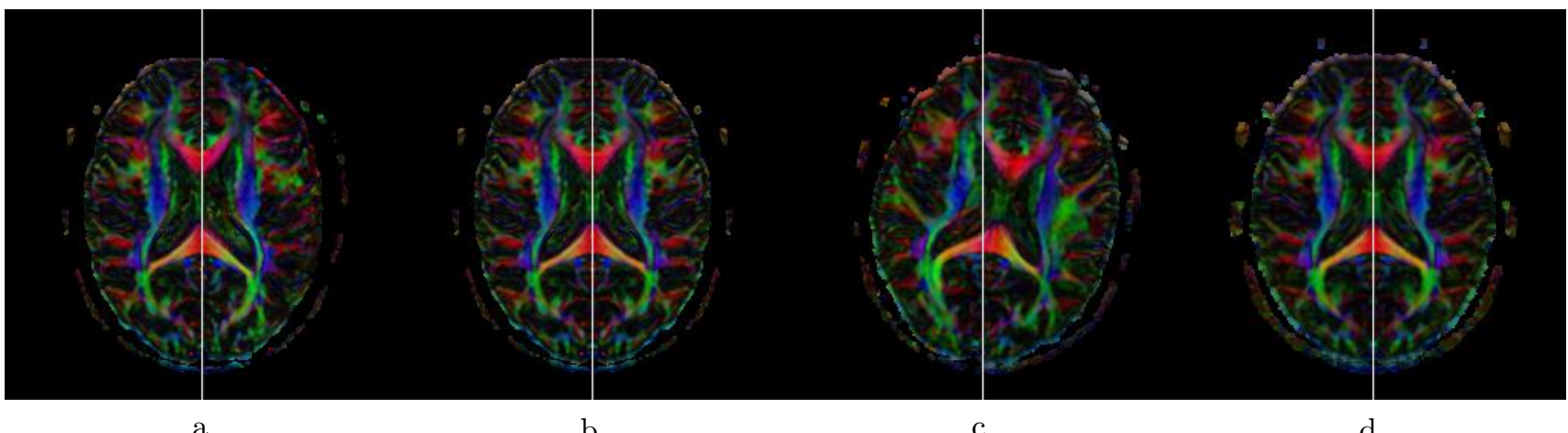

a

$\mathrm{b}$

d

Figure 1. Synthetic experiments. In a real DTI (matrix size $=256 \times 256$, field of view $=22.4 \mathrm{~cm}$, number of slices $=$ 60 , slice thickness $=2 \mathrm{~mm}$ ), the mid-sagittal plane of the brain is manually aligned with the centre of the image grid (a). Half of the image is then removed and replaced by the flipped version of the other half (b). In this new image, the central plane of the grid is a perfect symmetry plane for the brain. This image is then deformed by a rigid transformation $R_{1}$ that moves each point $p$ to $p^{\prime}$ following: $p^{\prime}=R_{1}(p)=\mathbf{R}_{\mathbf{x}}\left(\phi_{x}\right) \mathbf{R}_{\mathbf{y}}\left(\phi_{y}\right) \mathbf{R}_{\mathbf{z}}\left(\phi_{z}\right) p+\mathbf{t}$, where $\mathbf{R}_{\mathbf{x}}$ (resp. $\mathbf{R}_{\mathbf{y}}$ and $\mathbf{R}_{\mathbf{z}}$ ) is a rotation around the $x$ - (resp. $y$ - and $z$-) axis and $\mathbf{t}=\left(t_{x}, t_{y}, t_{z}\right)$ is a translation (c). We then apply our algorithm to compute the transformation $R_{2}$ correcting the tilt (d). The three parameters $\phi_{x}, t_{y}$ and $t_{z}$ leave the symmetry plane unchanged; they are set to 0 . We lead 400 different experiments, with random parameters $\phi_{y}, \phi_{z}$ and $t_{x}$ between $\pm 20^{\circ}$ and $\pm 20 \mathrm{~mm}$ respectively.
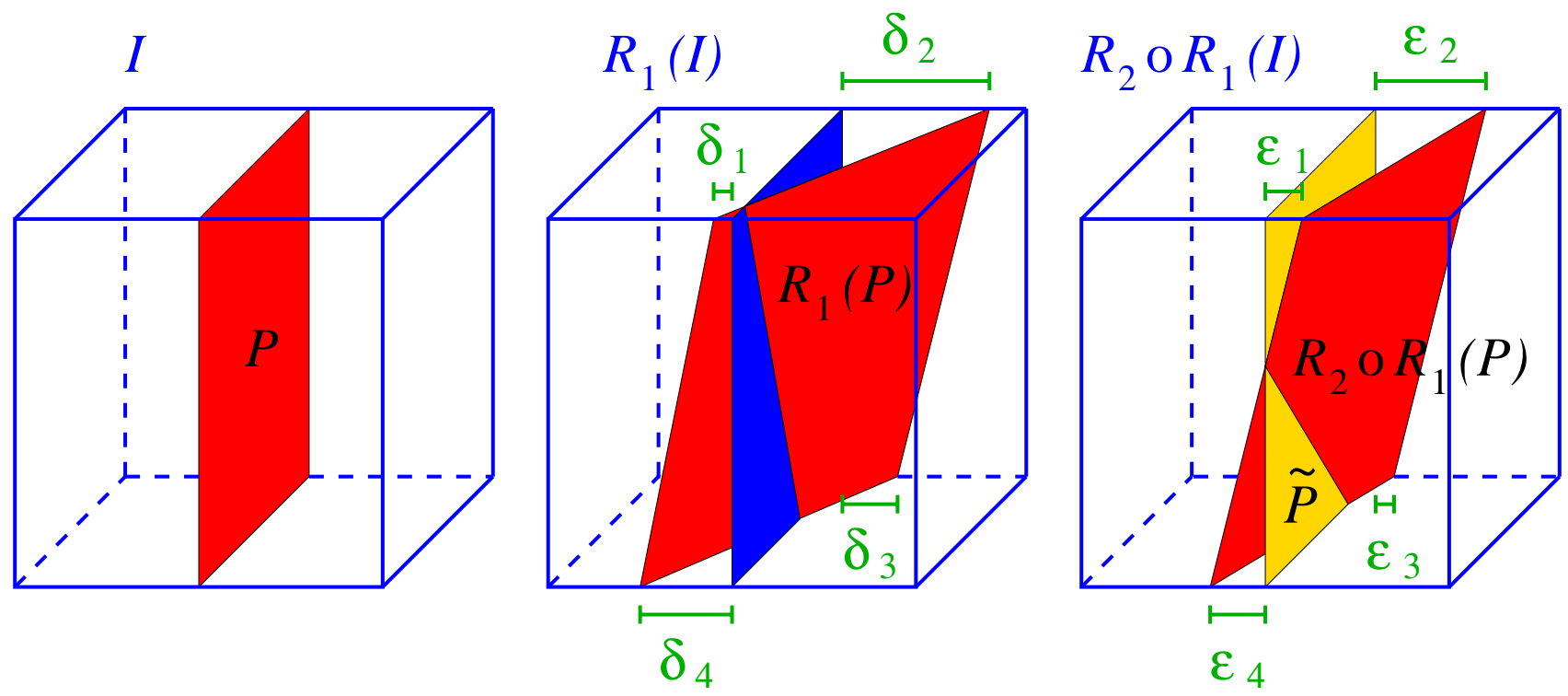

Figure 2. Robustness and accuracy of the algorithm: $\delta=\max \left(\delta_{i}\right)$ measures its capture $\operatorname{range}$, whereas $\epsilon=\max \left(\epsilon_{i}\right)$ measures its accuracy. 


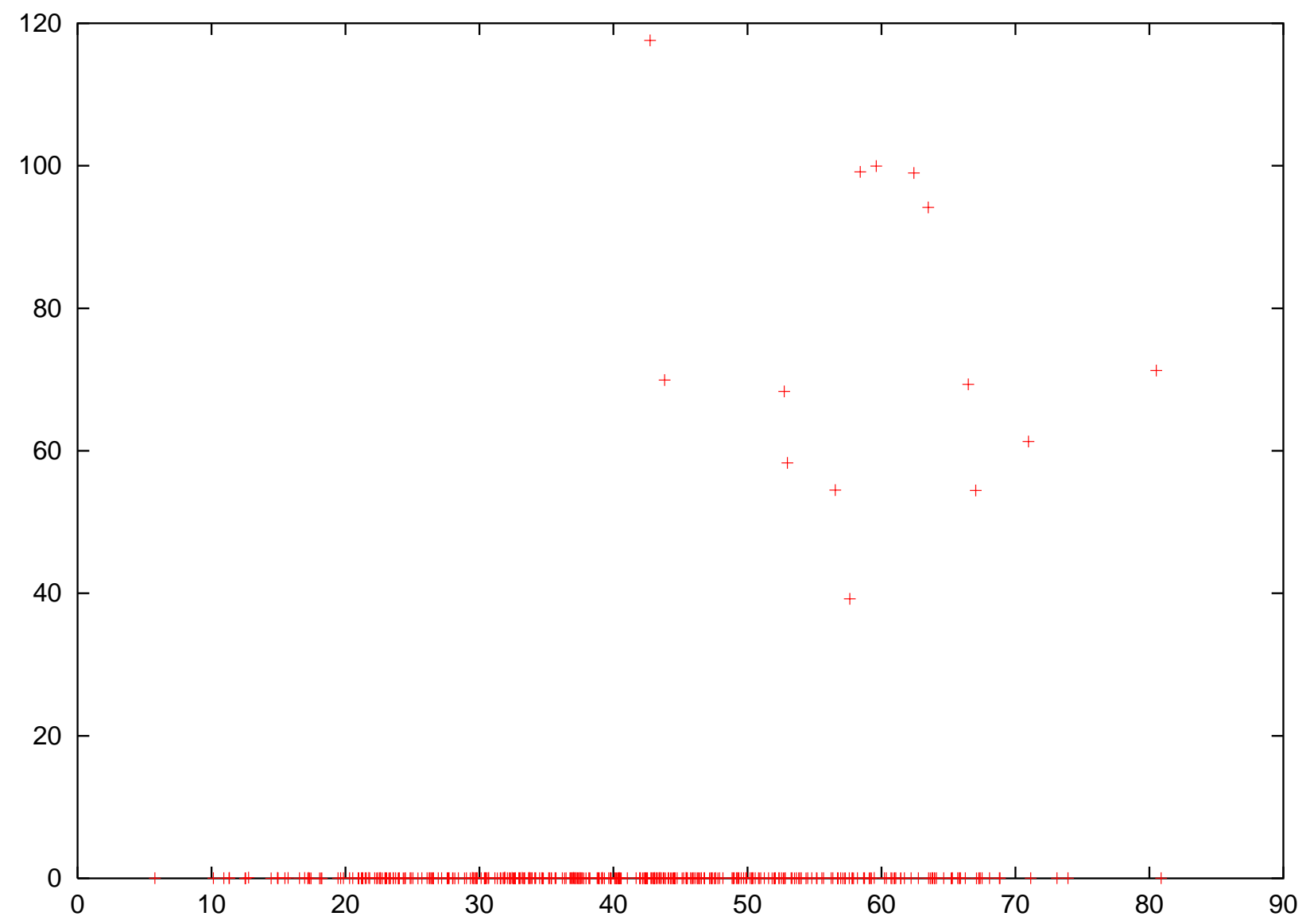

Figure 3. Plot of the robustness/accuracy results for the $\mathbf{4 0 0}$ random experiments: horizontal axis $=\delta$ and vertical axis $=\epsilon$; the algorithm never fails when $\delta<43 \mathrm{~mm}$. The first error occurs for an experiment where $\delta=43.82$ $\mathrm{mm}$, which corresponds to $\left(t_{x}, \phi_{y}, \phi_{z}\right)=(-18.26,18.45,2.68)$.
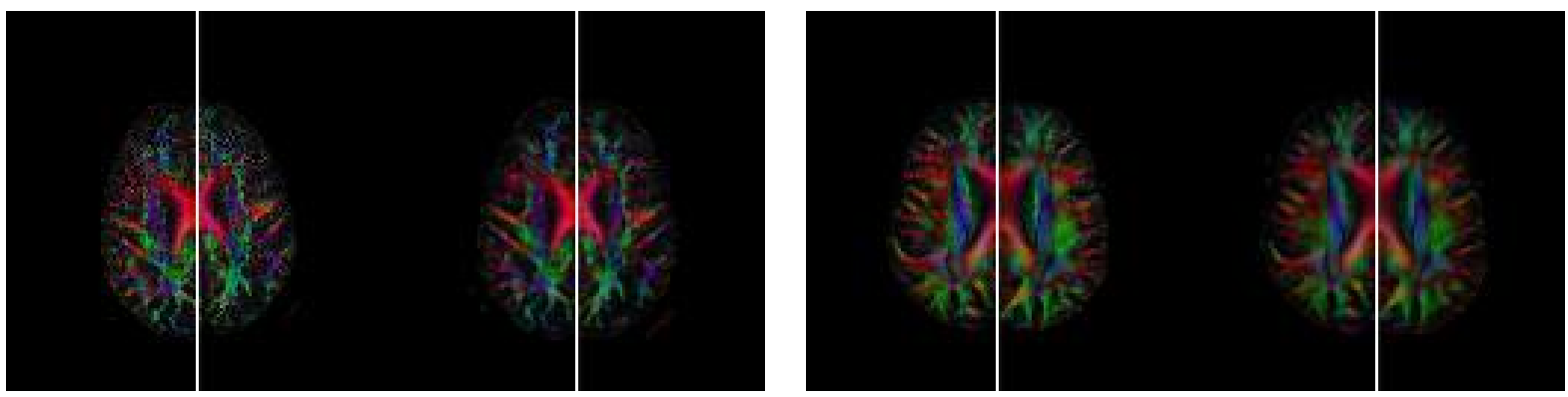

Figure 4. Results on real DTI data. Two patients with Parkinson's disease (left: original image and right: realigned image). The color coding is: red for left-right, green for antero-posterior and blue for top-bottom. It is applied to the principal diffusion of direction (eigenvector of the diffusion tensor with the largest eigenvalue), with the brightness proportional to the fractional anisotropy (FA). 